\title{
RECEIVER WIDELANE ANALYSIS AND ITS EFFECT ON PRECISE POINT POSITIONING
}

\author{
Mohamed Elsobeiey \\ Department of Hydrographic Surveying, Faculty of Maritime Studies, King Abdulaziz University \\ P.O. Box 80401 Jeddah 21589, Saudi Arabia \\ elsobeiey@gmail.com
}

KEY WORDS: Precise Point Positioning, Widelane Bias, Hardware Delay, Ambiguity Resolution

\begin{abstract}
:
Typically, differential carrier-phase-based methods have been used in positioning applications that require high accuracy. The main advantage of differential methods is solving the carrier-phase ambiguities and obtain millimetre-level accuracy carrier-phase measurements. Recent studies showed that it is possible to fix the un-differenced carrier-phase ambiguities into integers which is well-known as un-differenced carrier-phase ambiguity resolution. Unfortunately, the IGS neglects satellite hardware delay during satellite clock corrections estimation process. In case of differential methods, however, this will not affect the user as all common errors between the reference and rover receivers will be cancelled out by. Point positioning, on the other hand, will be affected by neglecting satellite hardware delays as those hardware delays will be lumped into the carrier-phase ambiguities destroying its integer nature. To solve this problem, satellite clock corrections must be estimated based on clock correction for each observable bases. The user, on the other hand, can form the ionosphere-free linear combination and divide and fix its two components, namely widelane and narrowlane. If both ambiguities are successfully fixed, few millimetres level of accuracy measurements are then obtained. In this paper, one month (December, 2013) of GPS data is used to study the receiver widelane bias, its behaviour over time, and receiver dependency are provided. It is shown that the receiver widelane bias is receiver dependent, stable over time for high-grade geodetic receivers. These results are expected to have a great impact on precise point positioning (PPP) conversion time and PPP carrierphase ambiguity resolution.
\end{abstract}

\section{INTRODUCTION}

Recently, precise point positioning (PPP) is introduced as an alternative to the classical differential methods. However, PPP suffers from the long convergence time that is required to obtain comparable level of accuracy to that can be obtained from differential methods. In addition, modelling all error sources is mandatory in PPP rather than differential methods, where all common errors are successfully cancelled (see e.g. Kouba (2009), Elsobeiey and El-Rabbany (2012), Elsobeiey and El-Rabbany (2013)). Between-satellites single-difference (BSSD) model can be used to cancel out receiver clock error, receiver hardware delay, and receiver initial phase bias (Elsobeiey and El-Rabbany, 2014). Both un-differenced and BSSD models can apply the international navigation GNSS services (IGS) products such as satellite orbits and clock corrections.

Decoupled clock model can be used to account for satellite hardware delay and satellite initial phase bias (Collins, 2008). Decoupled clock corrections can be applied to either the undifferenced PPP models (Collins, 2008; Collins et al., 2010; Shi and Gao, 2010) or to BSSD models to achieve faster convergence time (Elsobeiey and El-Rabbany, 2014). The decoupled clock model includes two different clock corrections, namely, code and carrier-phase clock corrections. In this case, the code clock correction is estimated the same known method in which one of the receiver clock should be fixed. However, the estimation of the satellite clock corrections from the carrierphase requires minimum constrain least-squares solution by arbitrary fix one ambiguity associated with each phase clock, less one, and fix one of the phase clocks as a network datum (Collins et al., 2010).

A network of reference stations can be used to solve for the carrier-phase ambiguities. The average values of the fractional cycle part of the real valued widelane and narrowlane ambiguities can be used as corrections for widelane and narrowlane real ambiguities from a single receiver (Ge et al., 2008). However, the success of this method depends mainly on the update rate of the widelane and narrowlane corrections.

CNES/CLS IGS analysis center started since November, 2009 to produce phase clock corrections that keeps the integer property of the carrier-phase measurements (Laurichesse et al., 2010). The dual-frequency GPS data are used firstly to fix the widelane ambiguities using the well-known MelbourneWübbena linear combination along with the estimation of satellite widelane bias (WSB). After fixed the widelane ambiguity, the ionosphere-free ambiguity is fixed on the undifferenced level and the corresponding satellite phase clocks are estimated. These satellite clock corrections keep the integer nature of the carrier-phase ambiguities (Laurichesse et al., 2010). A user of single receiver can use the published WSBs to fix his widelane ambiguities and apply the CNES/CLS satellite clock corrections in the un-differenced level to solve for the narrowlane ambiguities.

In this paper, we present the results of receiver widelane analysis using long sessions of IGS Multi-GNSS Experiment (MGEX) stations of different kinds of GNSS receivers. The stability of receiver widelane bias is studied and its applications are described. 


\section{OBSERVATION EQUATIONS}

Observation equations of GPS pseudorange and carrier-phase measurements can be described as follows (Laurichesse et al., 2009):

$$
\begin{aligned}
& P_{1}=D_{1}+\Delta h_{P}+\left(I_{1}+\Delta \tau_{P}\right) \\
& P_{2}=D_{2}+\Delta h_{P}+\gamma\left(I_{1}+\Delta \tau_{P}\right) \\
& \lambda_{1} L_{1}=D_{1}+\lambda_{1} W+\Delta h-\left(I_{1}+\Delta \tau\right)-\lambda_{1} N_{1} \\
& \lambda_{2} L_{2}=D_{2}+\lambda_{2} W+\Delta h-\left(I_{1}+\Delta \tau\right)-\lambda_{2} N_{2}
\end{aligned}
$$

where $\quad P_{1}, P_{2}=$ pseudorange measurements on $\mathrm{L}_{1}$ and $\mathrm{L}_{2}$, respectively

$L_{1}, L_{2}=$ carrier-phase measurements on $\mathrm{L}_{1}$ and $\mathrm{L}_{2}$, respectively

$\lambda_{1}, \lambda_{2}=$ carrier-phase wavelengths of $\mathrm{L}_{1}$ and $\mathrm{L}_{2}$, respectively

$N_{1}, N_{2}=$ carrier-phase $\mathrm{L}_{1}$ and $\mathrm{L}_{2}$ ambiguities, respectively

$D_{1}, D_{2}=$ geometric distance between satellite antenna phace center to receiver phase center at frequencies $f_{1}$ and $f_{2}$, respectively, including tropospheric delay and other corrections

$W \quad=$ phase wind-up correction (cycles)

$I_{1} \quad=$ ionospheric delay on $\mathrm{L}_{1}$

$\Delta h=h^{r}-h^{s}=$ the difference between carrier-phase receiver and satellite clocks

$\Delta h_{P}=h_{P}^{r}-h_{P}^{s}=$ the difference between pseudorange receiver and satellite clocks

$\Delta \tau=\tau^{r}-\tau^{s}=$ the difference between phase clock at $f_{1}$ and ionosphere-free phase clocks

$\Delta \tau_{P}=\tau_{P}^{r}-\tau_{P}^{s}=$ the difference between pseudorange clock at $f_{1}$ and ionosphere-free pseudorange clocks (time group delay in $\mathrm{m}$ )

$$
\gamma=f_{1}^{2} / f_{2}^{2}
$$

Equations 1 through 4 are based on one clock for each observable and different reference clocks for code and carrier phase along with the corresponding offsets (Laurichesse et al., 2010).

\section{SATELLITE WIDELANE BIAS}

Widelane satellite biases $\mu^{s}$ can be identified using the following equation (Laurichesse et al., 2013):

$$
\langle\tilde{N}\rangle=N_{w}+\mu^{r}-\mu^{s}
$$

where $\quad N_{w}=$ Melbourne-Wübbena linear combination

$\mu^{r}=$ receiver widelane bias

\langle\rangle means the average value over satellite pass (in case of no cycle slip)
CNES/CLS IGS analysis center estimates the daily satellite widelane biases using network of IGS stations and the WSB file is available at ftp://ftpsedr.cls.fr/pub/igsac/ . Figure 1 shows the daily satellite WSBs since 2009.

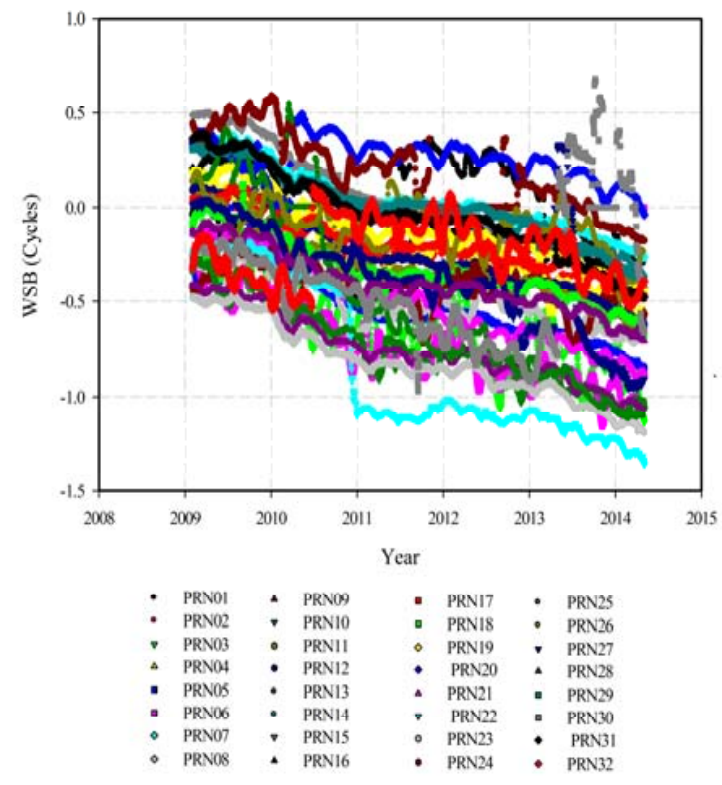

Figure 1. CNES/CLS Daily WSB Since 2009

As seen in Figure 1, WSBs are very stable and any jumps are due to satellite replacement. The available WSBs may be applied by a user of single receiver to estimate the receiver wide lane bias using Equation 5.

\section{RECEIVER WIDELANE BIAS}

Using the published values of WSBs, receiver widelane bias (RWB) can be estimated epoch by epoch and averaged over specific time window. In this study, we estimated the RWB as a weighted daily average using satellite elevation angle dependent weighting scheme. Two IGS-MGEX stations are chosen such that they are occupied with two different kind of receivers Figure 2.

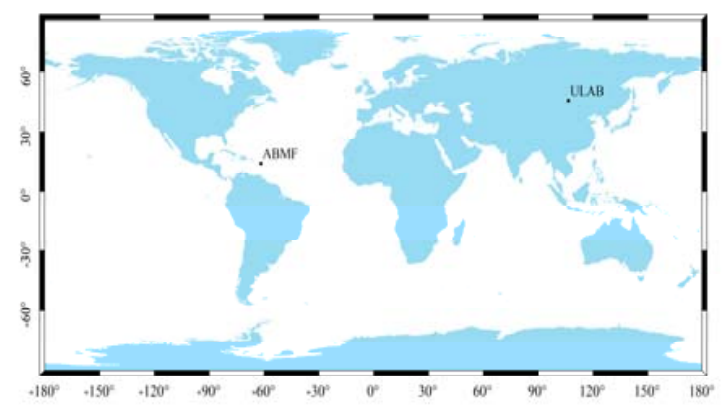

Figure 2. ABMF and ULAB IGS-MGEX Stations

Equation 5 can be re written as:

$\mu^{r}=\langle\tilde{N}\rangle-N_{w}+\mu^{s}$ 
For one station, to ensure consistency between all satellites arcs, the value of RWB can be adjusted by +1 or -1 to keep all values close to each other in addition to removing any outliers. The values of $\mu^{r}$ for both stations are shown in Figures 3 and 4 , respectively.

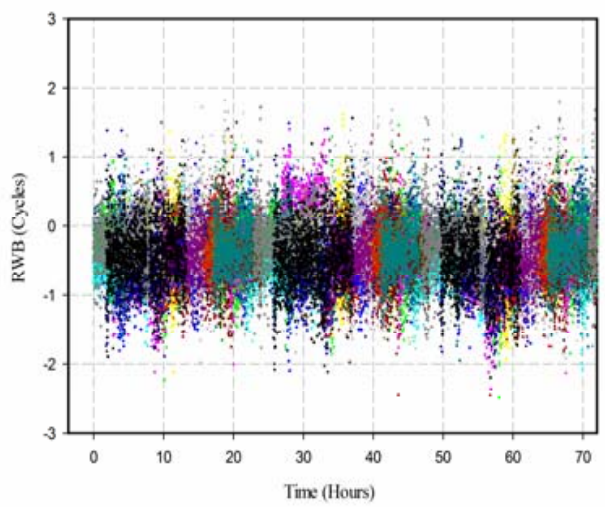

Figure 3. Three Days of RWB at ABMF IGS-MGEX Station

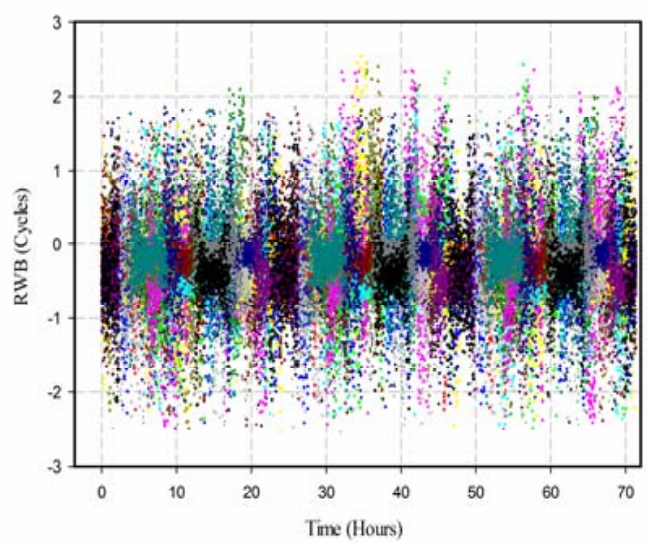

Figure 4. Three Days of RWB at ULAB IGS-MGEX Station

As seen in Figures 3 and 4, the computed RWBs are contaminated by the code noise and multipath. In addition, based on each receiver environment, the data quality is different and hence we notice that RWB at ULAB station is noisier than that at ABMF station. Figures 5 shows the daily average of one month of processing during December, 2013.

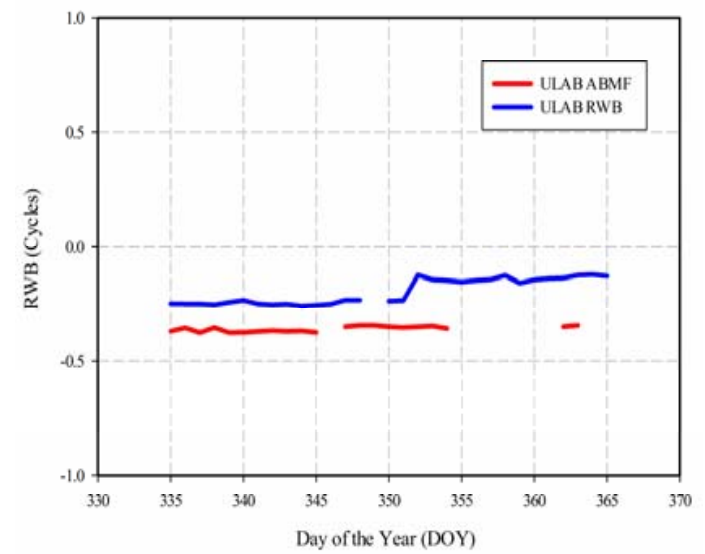

Figure 5. Daily RWB of Both ABMF and ULAB IGS-MGEX Stations
As seen in Figure 5, the RWB is receiver dependent and can be considered stable for short period of time for high grade geodetic receiver. Also, the noise in ULAB station data affects the estimated RWB causing some fluctuations. The reason of the jump in RWB at ULAB receiver is not clearly known. May be due to receiver reset or some other reasons.

\section{CONCLUSIONS}

In this paper, a comprehensive study on receiver widelane bias is carried out. GPS data from two IGS-MGEX stations, namely ABMF and ULAB, are used to compute the well-known Melbourne-Wübbena linear combination during a session extends to one month (during December, 2013). The widelane ambiguities are averaged and removed from MelbourneWübbena combination leaving both satellite/receiver widelane bias. The satellite widelane biases obtained from CNES/CLS IGS analysis centre are applied to obtain a data series represent the receiver widelane bias. According to each satellite elevation angle, a weighted average of the receiver widelane bias are estimated on daily basis. It is shown that receiver widelane bias is receiver type dependent and can be considered stable for high-grade geodetic receiver. These results are promising especially when applied to carrier-phase ambiguity resolution in the un-differenced PPP models.

\section{ACKNOWLEDGMENTS}

The data sets used in this research were obtained from the IGS Multi-GNSS Experiment (MGEX) website http://igs.org/mgex. Satellite widelane biases applied in this paper are obtained from CNES/CLS IGS analysis centre ftp://ftpsedr.cls.fr/pub/igsac/

\section{REFERENCES}

Collins, P., 2008. Isolating and Estimating Undifferenced GPS Integer Ambiguities, Proceedings of the 2008 National Technical Meeting of The Institute of Navigation, San Diego, CA, pp. 720-732.

Collins, P., Bisnath, S., Lahaye, F., Heroux, P., 2010. Undifferenced GPS Ambiguity Resolution Using the Decoupled Clock Model and Ambiguity Datum Fixing. NAVIGATION, Journal of The Institute of Navigation, Vol. 57, No. 2, Summer 2010, pp. 123-135.

Elsobeiey, M., El-Rabbany, A., 2012. On Modelling of SecondOrder Ionospheric Delay for GPS Precise Point Positioning. The Journal of Navigation 65, 59-72.

Elsobeiey, M., El-Rabbany, A., 2013. Rigorous Analysis of Modernized GPS C5 Differential Hardware Delay, The 2013 Canadian Institute of Geomatics Annual Conference and the 2013 International Conference on Earth Observation for Global Changes (EOGC'2013), Toronto, Ontario, Canada, pp. 159162.

Elsobeiey, M., El-Rabbany, A., 2014. Efficient BetweenSatellite Single-Difference Precise Point Positioning Model. Journal of Surveying Engineering 140, 04014007. 
Ge, M., Gendt, G., Rothacher, M., Shi, C., Liu, J., 2008. Resolution of GPS carrier-phase ambiguities in Precise Point Positioning (PPP) with daily observations. J Geod 82, 389-399.

Kouba, J., 2009. A guide to using International GNSS Service (IGS) products. Available online at http://igscb.jpl.nasa.gov/igscb/resource/pubs/GuidetoUsingIGS Products.pdf, accessed on 17 Nov, 2013.

Laurichesse, D., Cerri, L., Berthias, J.P., Mercier, F., 2013. Real time precise GPS constellation and clocks estimation by means of a Kalman filter. Proceedings of the ION GNSS 2013, September 16-20, 2013 - Nashville, Tennessee.

Laurichesse, D., Mercier, F., Berthias, J.P., Broca, P., Cerri, L., 2009. Integer Ambiguity Resolution on Undifferenced GPS Phase Measurements and its Application to PPP and Satellite Precise Orbit Determination. Navigation, Journal of the institute of Navigation Vol. 56, Nㅜ2, Summer 2009.

Laurichesse, D., Mercier, F., Berthias, J.P., Broca, P., Cerri, L., 2010. Real-Time PPP with undifferenced integer ambiguity resolution,experimental results. Proceedings of the ION GNSS 2010, September 2010, Portland, Oregon.

Shi, J., Gao, Y., 2010. Analysis of the Integer Property of Ambiguity and Characteristics of Code and Phase Clocks in PPP using a Decoupled Clock Model. Proceedings of the 23rd International Technical Meeting of The Satellite Division of the Institute of Navigation (ION GNSS 2010), Portland, OR, September 2010, pp. 2553-2564. . 